\title{
Pachytene piRNAs instruct massive mRNA elimination during late spermiogenesis
}

Lan-Tao Gou, Peng Dai, Jian-Hua Yang, Yuanchao Xue, Yun-Ping Hu, Yu Zhou, Jun-Yan Kang, Xin Wang, Hairi Li, Min-Min Hua, Shuang Zhao, Si-Da Hu, Li-Gang Wu, Hui-Juan Shi, Yong Li, Xiang-Dong Fu, Liang-Hu Qu, En-Duo Wang, Mo-Fang Liu

Cell Research (2015) 25:266. doi:10.1038/cr.2015.14; published online 3 February 2015

Addendum to: Cell Research (2014) 24:680-700. doi:10.1038/cr.2014.41; published online 2 May 2014

The RIP, CLIP, and RNA-seq data in this paper can be download via the following link. (SRA accession number: SRP043638)

http://www.ncbi.nlm.nih.gov/Traces/sra/?study=SRP043638

The microarray data can be download via the following link. (GEO accession number: GSE59291)

http://www.ncbi.nlm.nih.gov/geo/query/acc.cgi?acc=GSE59291 